Check for updates

Cite this: RSC Adv., 2019, 9, 476

Received 3rd November 2018

Accepted 19th December 2018

DOI: 10.1039/c8ra09106d

rsc.li/rsc-advances

\section{A facile synthesis of 1,3,6,8-pyrenesulfonic acid tetrasodium salt as a hydrosoluble fluorescent ink for anti-counterfeiting applications $\uparrow$}

\author{
Liangzhe Chen, (D) Biao Hu, Jieyu Zhang, (D) Jinmei Zhang, Shuting Huang, Ping Ren, \\ Yang Zou, Fuyuan Ding, (ID Xinghai Liu (D) and Houbin Li (iD *
}

In this work, 1,3,6,8-pyrenesulfonic acid sodium salt (PTSA) was successfully synthesized via a one-step sulfonating reaction. This method is more convenient, effective and eco-friendly than the traditional one. The as-prepared PTSA exhibits pure blue fluorescence under UV light. Due to its excellent fluorescent properties and water solubility, PTSA was used to prepare water-soluble invisible inks based on hydroxyethyl cellulose (HEC) aqueous solution. Notably, the resulting inks possessed acceptable stability after being stored for 30 days. Besides, the red/green/blue fluorescent inks were obtained by adding extra pigments, all of which exhibited excellent rheology and thixotropy properties. Subsequently, various patterns, including a QR code, the logo of Wuhan University, Chinese characters and so on, were printed on non-background paper through ink-jet and screen printing, and the as-prepared materials exhibited good water solubility and outstanding fluorescence performances, indicating that the fluorescent PTSA material is a promising candidate for anti-counterfeiting applications.

\section{Introduction}

Anti-fake labels are extensively used in ID cards, bills, luxury items, and goods, and have become more and more important for public safety. ${ }^{1}$ Traditional anti-counterfeiting technologies and materials are expensive, complex and can be easily copied. Hence, it is a significant challenge to develop a kind of anticounterfeiting label that is cost-effective and hard to duplicate.

Water-based inks can be printed on substrates as anticounterfeiting labels, which are free of organic solvent. Due to their eco-friendliness, they have aroused wide attention. Traditional water-based anti-fake materials consist of rare-earth (Er, Tb, Y et al. $)^{2-4}$ or other nanophase materials (Au, Ag, CQDs et al.), ${ }^{5-8}$ which not only involve harsh conditions during the preparation process but are vulnerable to duplication.

To meet the above challenges, the organic materials ${ }^{9,10}$ based on fluorescence process have wide applications as luminescent inks, which are featured by longer-wavelength visible emission when absorbing shorter-wavelength excitation, mostly ultraviolet (UV) light. The luminescent materials can be made into invisible inks,$^{11}$ which show the confidential information when exposed to UV light. Moreover, the luminescent materials can be formulated to generate colourful fluorescence inks by mixing various extra pigments. However, the lipophilicity of organic

School of Printing and Packaging, Wuhan University, Wuhan 430072, P. R. China. E-mail:lhb@whu.edu.cn

$\dagger$ Electronic supplementary information (ESI) available. See DOI: $10.1039 / \mathrm{c} 8 \mathrm{ra0} 9106 \mathrm{~d}$ fluorescence materials would limit its wide application. ${ }^{11}$ So selecting a type of organic material and modifying it to acquire hydrophilic property is of great importance.

Pyrene possesses extended $\pi$-bond and rigid plane structure which owns unique fluorescent properties, such as longer fluorescent life, better luminescence stability, extremely response to micro-environment and so on. ${ }^{12}$ Due to the excellent performance, pyrene and its derivatives have been extensively used in various areas. ${ }^{13,14}$ To date, some functional groups such as sulfonic acid groups $\left(-\mathrm{SO}_{3} \mathrm{H}\right),{ }^{15}$ formyl groups $(-\mathrm{CHO})^{16}$ and other groups ${ }^{17}$ have been introduced to pyrene structure to improve its solubility. Among them, it's more efficient to bring $-\mathrm{SO}_{3} \mathrm{H}$ group into pyrene ring to improve its water solubility.

The traditional synthesis of PTSA was a two-step reaction ${ }^{18,19}$ shown in Fig. 1a. Firstly, the pyrene-1-sulfonic acid sodium salt (PyS) was synthesized by sulfonating. ${ }^{20}$ Then, the as-prepared PyS was further sulfonated by oleum to obtain PTSA. The twostep synthesis method needs lots of separation and purification procedures which would cause environment damage and raw materials waste.

Hence, a novel and facile one-step method for synthesizing PTSA is proposed and the synthesis procedure is displayed in Fig. $1 \mathrm{~b}$ and Scheme 1. PTSA can be synthesized via sulfonating using $50 \%$ oleum, which is more facile, cost effective and has less impact on environment. Subsequently, the structure analysis is performed to prove the successful synthesis of PTSA, and the optical properties of PTSA are also investigated, which displays pure blue fluorescence under UV light. In 
(a)

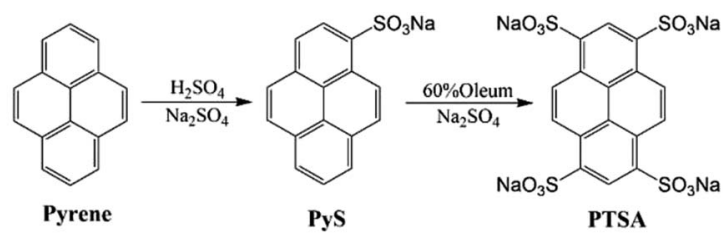

(b)

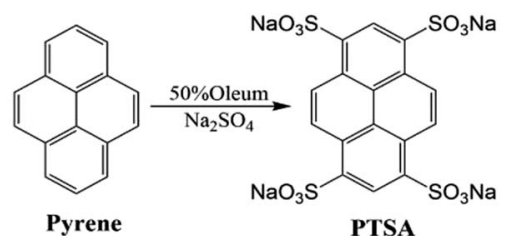

Fig. 1 (a) Traditional synthesis procedure of PTSA. (b) One-step synthesis process of PTSA.

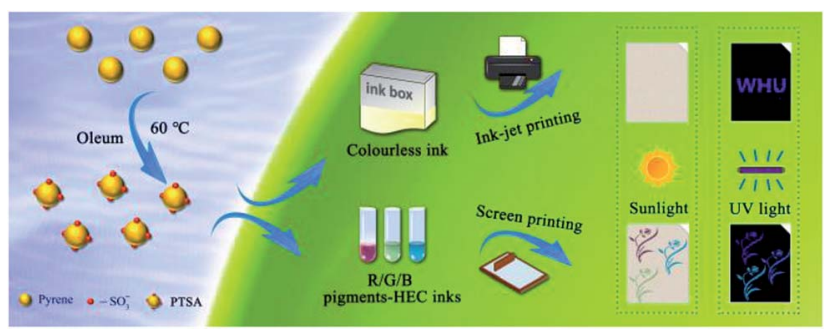

Scheme 1 A schematic of the synthesis procedure of PTSA and the anti-fake application.

addition, we utilize ink-jet printing to verify the feasibility of prepared inks containing a certain amount of PTSA and HEC, which only show secret information under UV light. Moreover, the red/green/blue $(\mathrm{R} / \mathrm{G} / \mathrm{B})$ printable inks are prepared by adding PTSA to HEC/pigments water solution, which are examined to possess good viscosity and thixotropic. Furthermore, various patterns are printed on non-background paper via screen printing technology, and the as-prepared materials exhibited good water solubility and outstanding fluorescence performances, demonstrating great potential of PTSA in multicolour anti-counterfeiting.

\section{Experimental section}

\subsection{Materials}

Pyrene (97\%), oleum (50\%), nitrobenzene (AR), calcium hydroxide (AR), sodium carbonate (AR), $n$-butanol (AR), sodium chloride (AR), chitosan and hydroxyethyl cellulose (HEC) were purchased from Sinopharm Chemical Reagent Co., Ltd and used as received. DISPercoll U54 was obtained from Bayer Co., Ltd and WPU-538 purchased from Jitian Chemical Reagent Co., Ltd. Pigments (red, green and blue) and the non-background paper free of fluorescer and other colours $(210 \times 297 \mathrm{~mm}$, $95 \mathrm{~g} \mathrm{~m}^{-2}$ ) were purchased from commerce. Deionized water was used during the experiment.

\subsection{Synthetic procedures of PTSA}

Scheme of one-step method to synthesize PTSA was shown in Fig. 1b. First, $3.5 \mathrm{~mL}$ of $50 \%$ fuming sulfuric acid was added in batches into the solution of pyrene $(1.01 \mathrm{~g}, 5 \mathrm{mmol})$ in nitrobenzene $(20 \mathrm{~mL})$. The mixture was stirred at $30{ }^{\circ} \mathrm{C}$ for $6 \mathrm{~h}$. The well-mixed materials were heated up to $60^{\circ} \mathrm{C}$ and kept stirring at a high speed for $12 \mathrm{~h}$ until a greenish yellow precipitate formed. Then, the resultant mixture was poured into ice water. After that, the calcium hydroxide slurry was added slowly to neutralize materials followed by filtration. Subsequently, the sodium carbonate was added in the filtrate. $n$-Butanol was used to extracted nitrobenzene from the mixture completely. Finally, the subnatant was treated with rotary evaporator at $60-80{ }^{\circ} \mathrm{C}$. The raw product was purified using saturated sodium chloride solution for recrystallizing to obtain the expected PTSA, as a yellow crystalline solid. ${ }^{1} \mathrm{H} \mathrm{NMR}\left(500 \mathrm{MHz}, \mathrm{d}_{6}-\mathrm{D}_{2} \mathrm{O}\right) \delta 9.170-$ $9.150(\mathrm{~s}, 4 \mathrm{H}) ; 9.150-9.130(\mathrm{~s}, 2 \mathrm{H}) .{ }^{13} \mathrm{C}$ NMR $\left(101 \mathrm{MHz}, \mathrm{D}_{2} \mathrm{O}\right)$ $\delta 136.83,128.78,126.98,125.39,124.57$. MS (ESI) $587.2\left[{\left.\mathrm{M}-\mathrm{Na}^{+}\right] .}^{+}\right.$

\subsection{Ink-jet printing process of the PTSA fluorescent ink}

The non-background paper was chosen as a printing substrate. The hydrosoluble PTSA materials were mixed with HEC solution to serve as a colourless ink. Then, the viscosity of ink was tested and the value was only $0.005 \mathrm{~Pa} \mathrm{~s}$, which is lower than the standard viscosity requirement of ink-jet printing ( $0.100 \mathrm{~Pa} \mathrm{~s})$, indicating that the prepared ink can be applied. Various patterns were printed by the EPSON L301 Printer. After the patterns were dried, the images can be displayed under irradiation of an UV (365 nm) analyser.

\subsection{Screen printing process of the $R / G / B$ fluorescent ink}

R/G/B fluorescent inks were formulated by adding PTSA to asprepared R/G/B pigments-HEC solution, respectively, where HEC acted as ink binder, increasing the viscosity of inks. The non-background paper was chosen as a substrate for printing. Screen printing was carried out using a mesh (300 mesh-counts) with different patterns. The three kinds of fluorescent inks were forced to the mesh and transferred onto the paper. The designs of different colours were dried under atmospheric environment and can be displayed by UV analyser.

\subsection{Viscosity under shear rate ramp}

The rheometer (KINEXUS PRO: Malvern, Shanghai, China) was used with parallel plate to clarify the viscosity of inks under shear rate ramp. The shear ramp ranged from 0.1 to $1000 \mathrm{~s}^{-1}$. The relationship between viscosity and shear is calculated using following equation: ${ }^{21}$

$$
\eta_{\mathrm{a}}(\gamma)=\tau(\gamma) / \gamma
$$

where $\eta_{\mathrm{a}}(\gamma)$ is viscosity, $\tau(\gamma)$ is shear stress, and $\gamma$ is shear rate.

\subsection{Rheological tests during printing process}

In the process of screen printing, the shear rate was changed constantly during the damage and recovery of ink structure. ${ }^{22}$ To examine the rheological properties during printing process, the printing simulation was implemented at $25{ }^{\circ} \mathrm{C}$ with $50 \%$ relative humidity. There were three process: first, the applied shear rate maintained at $0.1 \mathrm{~s}^{-1}$ for $30 \mathrm{~s}$, which was close to the 
situation before operation; second, the shear rate was increased to $1000 \mathrm{~s}^{-1}$ for $60 \mathrm{~s}$, which was similar to the printing speed during printing; third, the shear rate fell to $0.1 \mathrm{~s}^{-1}$ for $120 \mathrm{~s}$, corresponding to the viscosity recovery.

\subsection{Measurement of colour parameters and optical density}

The optical density was collected from a CH8105 GretagMacbeth SpectroEye. Colour parameters of samples were measured on an Xrite SP64 colour meter. $L$ (lightness), $a$ (redness-greenness) and $b$ (yellowness-blueness) were obtained to evaluate the property of colour. The total colour difference $(\Delta E)$ was calculated as follows:

$$
\Delta E=\left(\Delta L^{2}+\Delta a^{2}+\Delta b^{2}\right)^{1 / 2}
$$

where $\Delta L=L-L_{0} ; \Delta a=a-a_{0} ; \Delta b=b-b_{0} . L_{0}, a_{0}, b_{0}$ are colour values under white light; $L, a, b$ are colour values under UV light.

\subsection{Characterisation}

FT-IR spectra were obtained on a Nicolet 5700 spectrometer. UV-Vis absorption spectra were carried out using a Shimadzu 3600 spectrophotometer. The fluorescent spectra was recorded on a Hitachi F-4600 5J2-0004 spectrophotometer with an additional CNI $(2 \mathrm{~W}) 980 \mathrm{~nm}$ laser. ${ }^{1} \mathrm{H}$ and ${ }^{13} \mathrm{C}$ nuclear magnetic resonance (NMR) spectra were recorded on a $400 \mathrm{MHz}$ Bruker Advance. Electrospray ionization mass spectrometry (MS) was used on a Finnigan LCQ advantage ESI-MS. Multicolour images under sunlight and UV irradiation were taken by using a Nikon D5200 camera with an UV-IR-cut filter. The ink-jet printing was performed with an EPSON L301 printer. The excitation light source for obtaining the fluorescence images was a ZF-20D UV analyser with three types of light $(254 \mathrm{~nm}, 365 \mathrm{~nm}$ and white light).

\section{Results and discussions}

FT-IR spectra was carried out in order to identify the structure of PTSA. The groups of PTSA and pyrene were examined as shown in Fig. S1. $\dagger$ Comparing curve a with b, the resemblance of peaks at $3036 \mathrm{~cm}^{-1}$ and $1625 \mathrm{~cm}^{-1}$ were assigned to the $\mathrm{C}-\mathrm{H}$ and $\mathrm{C}=\mathrm{C}$ bonds stretching vibration of benzene, respectively. The broad band in the region of $3400 \mathrm{~cm}^{-1}$ was attributed to the stretching vibration of $\mathrm{O}-\mathrm{H}$ bonds by residual adsorbed water. The successful grafting was confirmed by the characteristic bands at $1203 \mathrm{~cm}^{-1}, 1173 \mathrm{~cm}^{-1}, 665 \mathrm{~cm}^{-1}$ and $591 \mathrm{~cm}^{-1}$ attributed to the $-\mathrm{SO}_{3} \mathrm{Na}$ group. ${ }^{23}$ Otherwise, the peaks at $750 \mathrm{~cm}^{-1}$ was ascribed to the para-substituted of the benzene. According to the above results, the sulfonic acid groups were successfully grafted in pyrene ring. This result was also confirmed by NMR and MS spectra (Fig. S2-S4†).

$\mathrm{S}^{+} \mathrm{O}_{3} \mathrm{H}$ was confirmed to be a kind of electrophilic reagent that can replace $-\mathrm{H}$ with $-\mathrm{SO}_{3} \mathrm{H}$ in pyrene ring, which ionized from concentrated sulfuric acid. Comparing with $\mathrm{H}_{2} \mathrm{SO}_{4}$, oleum owns great mass of $\mathrm{SO}_{3}$, which possesses stronger ability to sulfonate..$^{24}$ It's the reason that PTSA can be synthesised with $50 \%$ oleum by one step method.
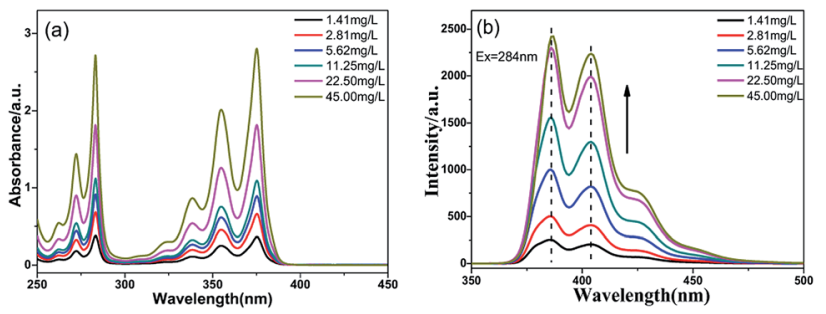

Fig. 2 (a) UV-Vis spectra and (b) fluorescence spectra of PTSA with concentrations ranging from 1.41 to $45.00 \mathrm{mg} \mathrm{L}^{-1}$ under UV light in $\mathrm{H}_{2} \mathrm{O}$.

The optical properties of PTSA in water were investigated by UV-vis spectra and Fluorescence spectra. As shown in Fig. 2a, the PTSA has two prominent absorption bands corresponding to the $\pi-\pi^{*}$ transition of pyrene monomer. ${ }^{25}$ Comparing the spectrum of pyrene in Fig. S5, $\dagger$ there are some red shifts from 272.6 to $284.0 \mathrm{~nm}$ and 335.0 to $375.8 \mathrm{~nm}$, respectively. It's due to the $-\mathrm{SO}_{3} \mathrm{Na}$ groups in pyrene ring reduce the energy of the excited state. Obviously, the intensity of bands increased without other bands appearing as the concentration increasing from 1.41 to $45.00 \mathrm{mg} \mathrm{L}^{-1}$, indicating that the stable structure of PTSA can against aggregation. ${ }^{23}$ In Fig. $2 \mathrm{~b}$, the hydrophilic PTSA showed strong blue fluorescence under $284 \mathrm{~nm}$ exciting light, with emission at 396 and $404 \mathrm{~nm}$.

Screen printing inks generally consist of pigments, binder, and solvent. Among them, binder plays an important role in protecting the pigment from damage by external force and facilitating the process of printing. ${ }^{22}$

In this paper, water was selected as solvent by reason of its eco-friendly character and simplicity, four kinds of watersoluble ink binders were blended with PTSA and the undissolved solid was separated through centrifugation. After being placed for $24 \mathrm{~h}$, the ink's appearance, $\mathrm{pH}$, stability, and fluorescence were measured (Table 1 and Fig. S6 $\dagger$ ).

In No. 3 ink, chitosan is strict with the solvent that can be dissolved in dilute acid, because of the free amino and hydroxyl. ${ }^{21}$ In consideration of the storage of ink, chitosan was not employed as ink binder. In No. 2 ink, some solids were observed after being placed for $12 \mathrm{~h}$, and the fluorescence decreased because of the white colour of DISPercoll U54. As an ink vehicle, WPU-538 showed good solubility and the appearance of No. 1 ink was translucent. Unfortunately, there is no thixotropic phenomenon in No. 1 and No. 2 ink, leading the ink to flow around in the process of printing. It's notable that after exposure in $365 \mathrm{~nm}$ light, No. 4 ink exhibited strong blue fluorescence while the fluorescence of No. 1 and No. 2 inks became weak and have a little shift. Therefore, HEC was suitable for ink binder due to the hydrophilic hydroxyl groups in submissive long chain can wrap pigments and help to produce a homogeneous ink. ${ }^{21}$ Moreover, there was no influence on fluorescence when HEC was added into inks, revealing that HEC was beneficial to show a refined structure during printing process.

As illustrated in previous literature, the rheological and thixotropic properties of screen printing inks are of vital factor for their application. ${ }^{26}$ To investigate the relationship between 
Table 1 Influence of different ink binders on PTSA ink's appearance and properties ${ }^{a}$

\begin{tabular}{|c|c|c|c|c|c|c|}
\hline No. & Binder & $\mathrm{H}_{2} \mathrm{O}:$ PTSA : binder (wt\%) & Appearance & Thixotropism & Stability & Impact on fluorescence \\
\hline 1 & WPU-538 & $90: 9: 1$ & Translucent & No & $>6$ months & No \\
\hline 2 & DISPercollU54 & $90: 9: 1$ & Milky & - & $12 \mathrm{~h}$ & Decrease \\
\hline 4 & HEC & $50: 0.5: 1$ & Transparent & Yes & $>6$ months & No \\
\hline
\end{tabular}

HEC and viscosity, we made an adjustment of the dosage with ink binder contents of 25,30 and $35 \mathrm{~g} \mathrm{~L}^{-1}$ in $\mathrm{R} / \mathrm{G} / \mathrm{B}$ inks, respectively. The inks were agitated strongly for $2 \mathrm{~h}$ to obtain the final stable inks.

The viscosity tests at different shear rate ranging from 0.1 to $1000 \mathrm{~s}^{-1}$ were shown in Fig. 3. We can find that the shear thinning thixotropic phenomenon arose in all inks. It's concluded that the viscosity of inks decreased as the shear rate increased. The reason was that the force between HEC and PTSA was broken by the increasing shear rate, resulting in an unstable ink system. Under the same mass percentage of PTSA, with the increase of the content of HEC, the viscosity of all inks augmented at the same shear rate. It was the result of the great mass percentage of HEC, giving rise to the augment of intermolecular force. ${ }^{21}$

During the screen printing process, ink's viscosity will decrease owing to the shearing of squeegee, which help ink transfer smoothly and homogeneously. ${ }^{27}$ Great mass percentage of HEC may cause the difficult movement of inks which will result in the jam of mesh. In contrast, small viscosity may lead to the sedimentation of pigment. Therefore, it's vital for us to select a suitable viscosity. In summary, $30 \mathrm{~g} \mathrm{~L}^{-1}$ of HEC is appropriate for screen printing. The viscosity of red ink: the maximum was $7.9 \mathrm{~Pa} \mathrm{~s}$ and the minimum was $0.4 \mathrm{~Pa} \mathrm{~s}$, green
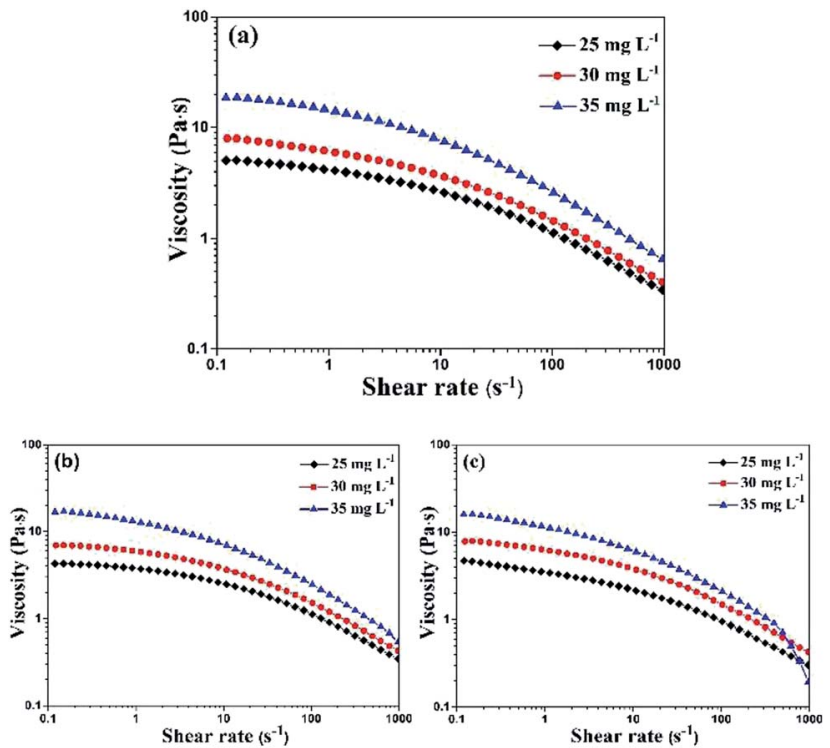

Fig. 3 Viscosity under shear rate ramp for PTSA inks with different HEC concentrations (a) red ink, (b) green ink and (c) blue ink. ink: the maximum 6.9 Pa s and the minimum 0.4 Pa s, blue ink: the maximum 7.7 $\mathrm{Pa} \mathrm{s}$ and the minimum 0.4 Pa s.

When the external force disappear, the viscosity of ink will increase and its mobility decreases, ensuring the inks fixing on the substrate. ${ }^{28}$ Thus, it's necessary to examine the rheological behaviour of inks using peak hold step (PHS) method at different shear rates in three intervals as shown in Fig. 4. In first interval, the shear rate was kept at $0.1 \mathrm{~s}^{-1}$ for $40 \mathrm{~s}$, then increased to $1000 \mathrm{~s}^{-1}$ for $30 \mathrm{~s}$, finally dropped to $0.1 \mathrm{~s}^{-1}$ and held for $120 \mathrm{~s}$ to facilitate the viscosity recovery.

It's clear that viscosity of all inks dropped rapidly when the shear rate changed from 0.1 to $1000^{-1}$, as a result of the damage of ink structure by shear stress, which was similar to the research of Wang et al. ${ }^{\mathbf{2 1 , 2 9}}$ After the shear rate declined to 0.1 $\mathrm{s}^{-1}$, the viscosity went up and the ink structure was restored. The viscosity of all inks could recover to its original level, whose recovery rates near $100 \%$. The recovery time was calculated as the time required for the viscosity of prepared ink to revert to the original. As shown in Table $\mathrm{S} 1, \uparrow$ the recovery time of green ink and blue ink were 45 and $47 \mathrm{~s}$, respectively, which was fitted for inks to dry and level on the substrate. Among them, red ink needs more time to resume, reaching $106 \mathrm{~s}$. It's likely that red pigment hindered the movement of inks because of its great structure, which should take more time for inks to rebuild the original structure.

As above, we have demonstrated that the PTSA possesses strong blue fluorescence under UV light and large-scale synthesis can be easily achieved by one-step method. The

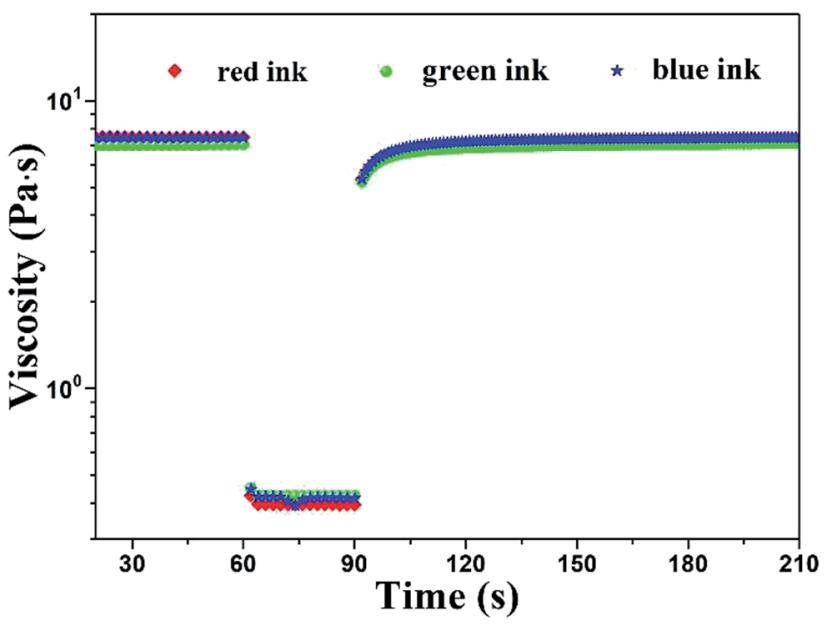

Fig. 4 Rheological behaviour of R/G/B inks during screen printing with $30 \mathrm{~g} \mathrm{~L}^{-1}$ of HEC. 


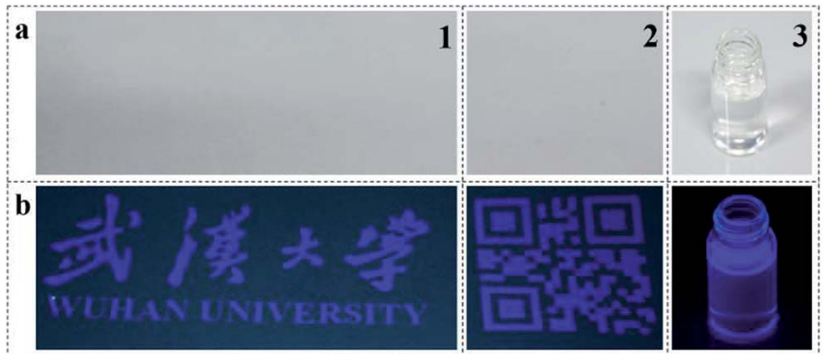

Fig. 5 Two anti-counterfeiting patterns and ink-jet printing inks on the non-background paper under (a) white light and (b) 365 nm UV light.

PTSA powder can facilely dissolve into aqueous solution, the colourless and stabilized solution is formed. Furthermore, the stability of ink was investigated after being placed at room temperature for 30 days. Obviously, the ink was transparent under sunlight, and blue fluorescence can be observed after being exposed in UV light (Fig. S7†). Surprisingly, it can still be printed on substrate and only displays the secret pattern under excitation light, indicating the prepared material is suitable for secure UV-to-visible printing. These result showed that the prepared inks can keep some secret information and be used for anti-fake technology. ${ }^{30}$

To verify this statement, PTSA inks were printed upon nonbackground paper via ink-jet method, the composition of colourless ink was displayed in Table S2. $\dagger$ Transparent and homogeneous aqueous solution (Fig. 5a3) with $3 \mathrm{~g} \mathrm{~L}^{-1}$ of PTSA was injected into the ink cartridge on an Epson printer, and it showed blue fluorescence under UV light (Fig. 5b3). As we can see, blue words and Chinese characters emerged under $365 \mathrm{~nm}$ UV lamp (Fig. 5b1). Besides, the inks were also adapted to other patterns, such as QR code (Fig. 5b2). The margin of the code was clear and the details were obvious. More importantly, we could not find any printed information on the surface of the paper (Fig. 5a1 and a2), and the patterns can be stored for several months. These results imply that the PTSA inks are suitable for anti-fake measures. ${ }^{31}$

In practical applications, colourful and designable patterns for anti-counterfeiting were difficult to simulate and duplicate. To demonstrate the suitability of PTSA inks for multi-coloured patterns, they were printed on the flexible non-background paper using screen printing, the composition of prepared inks could be found in Table S2. $\dagger$

The diverse patterns and R/G/B inks under white light and $365 \mathrm{~nm}$ UV light were presented in Fig. 6(I) and (II), respectively. Under white light, we can see that the red (Fig. 6(I)a4), green (Fig. 6(I)b4) and blue (Fig. 6(I)c4) inks were vivid and uniform. After exciting by $365 \mathrm{~nm}$ UV light, they show different colours due to the overlay of previous tints and fluorescence. ${ }^{32}$ The colour of red ink changed to amaranth (Fig. 6(II)a4), while the green ink switched to jade green (Fig. 6(II)b4) and the blue ink became baby blue (Fig. 6(II)c4). It's of great significance to observe that three types of patterns with different colours appeared on non-background paper, including four-leaf clover

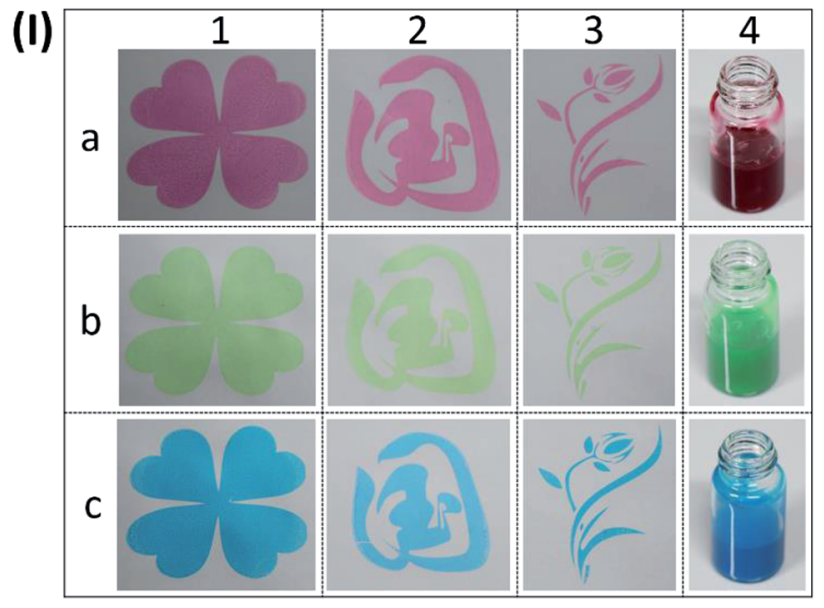

(II)

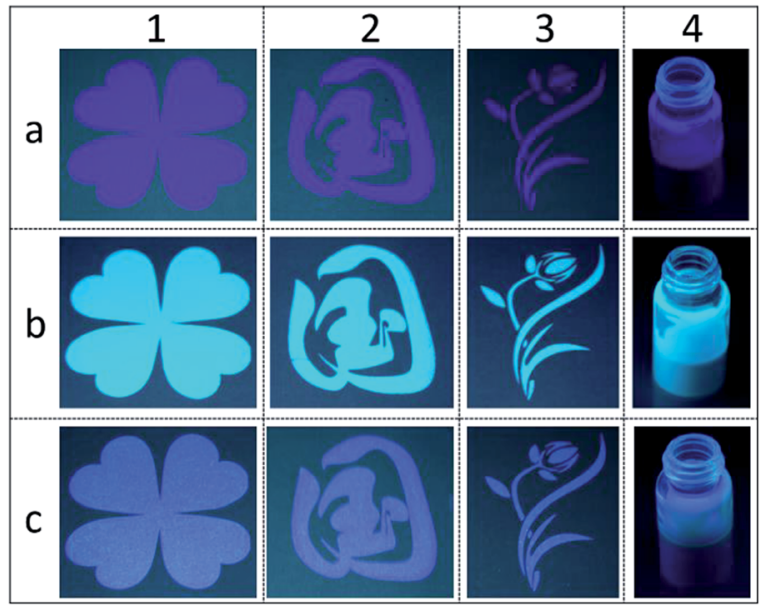

Fig. 6 Multicolour graphs of screen-printing patterns and R/G/B inks on the non-background paper under white light (I) and the $365 \mathrm{~nm}$ UV light (II). Screen printing patterns of (a) red inks, (b) green inks, and (c) blue inks.

(Fig. 6(I)1), Chinese characters (Fig. 6(I)2), and flower (Fig. 6(I) 3). After the $365 \mathrm{~nm}$ UV light excitation, the fluorescence patterns with different colours emerged, which was similar to their own colour under sunlight (Fig. 6(II)1, (II)2 and (II)3). We can find the subtle and clear margin of designable and multicoloured patterns, which indicates the as-prepared R/G/B inks are suitable for screen printing technology.

To further study the optical density and colour parameters of above printing samples, the optical density, $L^{*}, a^{*}, b^{*}$ and $\Delta E$ are presented in Table S3. $\dagger$ The optical density was an assessment of the thickness of dried ink layer. The highest value of optical density was observed for green ink, which could be relative to the wettability of paper substrate and the distribution of PTSA inks in the printing unit. ${ }^{33}$ It's obvious that the value of $\Delta E$ of screen printing samples after being exposed to UV light were greater than 4 , indicating that the differences were visible to the naked eye. However, the sample of ink-jet printing was colourless and unable to be detected, but we can find that the colour change in Fig. 5 was evident under 365 nm UV light. ${ }^{34}$ 


\section{Conclusions}

In summary, we reported a convenient, low-cost, and highthroughput organic fluorescence material for invisible and visible anti-fake and security application based on water-soluble inks. PTSA was successfully synthesized by sulfonic reaction via one-step method, which was more eco-friendly and straightforward than traditional procedure. The evaluation of optical property displayed that the as-prepared sample was a blue fluorescence material. Subsequently, HEC was employed as ink binder, which can protect the pigments and increase ink's viscosity. The colourless ink containing PTSA and HEC aqueous solution was coated on paper, which can be detected under $365 \mathrm{~nm}$ UV light while nothing appears under white light. Moreover, R/G/B inks were prepared by mixing PTSA with HEC/ pigment solution. The results of viscosity test showed that $30 \mathrm{~g}$ $\mathrm{L}^{-1}$ of HEC was suitable for inks, which displayed good thixotropic behaviour at the same time. When the as-prepared stable $\mathrm{R} / \mathrm{G} / \mathrm{B}$ inks were used to print various patterns via screen printing, these patterns could show pigment's colours under white light, and the fluorescence could be detected under $365 \mathrm{~nm}$ UV light. These water-soluble fluorescence materials could be a promising candidate for anti-counterfeiting label.

\section{Conflicts of interest}

There are no conflicts to declare.

\section{Acknowledgements}

This work was supported by NSAF (Grant No. U1830127). We would like to thank Large-scale Instrument and Equipment Sharing Foundation of Wuhan University for the support.

\section{Notes and references}

1 P. K. Kanika, S. Singh and B. K. Gupta, Chemistry, 2017, 23, 17144-17151.

2 M. Li, W. Yao, J. Liu, et al., J. Mater. Chem. C, 2017, 5, 65126520.

3 P. Kumar, K. Nagpal and B. K. Gupta, ACS Appl. Mater. Interfaces, 2017, 9, 14301-14308.

4 X. Zhou, Q. Ma, W. Yu, et al., J. Mater. Sci., 2015, 50, 78847895.

5 C. Campos-Cuerva, M. Zieba, V. Sebastian, et al., Nanotechnology, 2016, 27, 095702.

6 M. P. Sk and A. Chattopadhyay, RSC Adv., 2014, 4, 31994.

7 C. Sun, Y. Zhang, S. Kalytchuk, et al., J. Mater. Chem. C, 2015, 3, 6613-6615.

8 Y. M. Guo, Y. Z. Chen, F. P. Cao, L. J. Wang, Z. Wang and Y. M. Leng, RSC Adv., 2017, 7, 48386-48393.

9 J. Wang, Y. Gao, J. Zhang and H. Tian, J. Mater. Chem. C, 2017, 5, 4571-4577.
10 J. Rull-Barrull, M. d'Halluin, E. Le Grognec and F. X. Felpin, J. Mater. Chem. C, 2017, 5, 5154-5162.

11 X. Chen, X. Jin, J. Tan, et al., J. Colloid Interface Sci., 2016, 468, 300-306.

12 P. Sonar, M. S. Soh, Y. H. Cheng, J. T. Henssler and A. Sellinger, Org. Lett., 2010, 12, 3292-3295.

13 N. Chakraborty, A. Chakraborty and S. Das, J. Lumin., 2018, 199, 302-309.

14 Y. Sagara, T. Mutai, I. Yoshikawa and K. Araki, J. Am. Chem. Soc., 2007, 129, 1520.

15 Z. Miskolczy, Y. Takahashi, N. Kobayashi, S. Nakabayashi, A. Loukanov and L. Biczók, Colloids Surf., A, 2018, 552, 161-168.

16 R. I. Khan, A. Ramu and K. Pitchumani, Sens. Actuators, B, 2018, 266, 429-437.

17 V. de Halleux, J. P. Calbert, P. Brocorens, et al., Adv. Funct. Mater., 2004, 14, 649-659.

18 D. M. Marmion, Handbook of US colourants: foods, drugs, cosmetics, and medical devices, John Wiley \& Sons, 3rd edn, 1991.

19 S. Jitian, S. R. White, H. H. Yang and A. Weisz, J. Chromatogr. A, 2014, 1324, 238-241.

20 F. M. Menger and L. G. Whitesell, J. Org. Chem., 1987, 52, 3793-3798.

21 H. Wang, T. Guo and H. Li, J. Appl. Polym. Sci., 2016, 133, 43997.

22 H. Wang, T. Guo, Y. Zhang, Q. Zhang and H. Li, Prog. Org. Coat., 2017, 111, 75-82.

23 X. Xie, W. Lei, X. Liu, et al., Mater. Lett., 2011, 65, 1234-1237. 24 A. R. Katritzky, M. S. Kim, D. Fedoseyenko, K. Widyan, M. Siskin and M. Francisco, Tetrahedron, 2009, 65, 11111114.

25 A. L. Costa, A. C. Gomes, M. Pillinger, I. S. Goncalves and J. S. Seixas de Melo, Langmuir, 2015, 31, 4769-4778.

26 J. Liang, K. Tong and Q. Pei, Adv. Mater., 2016, 28, 59865996.

27 S. M. P. Meroni, Y. Mouhamad, F. De Rossi, et al., Sci. Technol. Adv. Mater., 2017, 19, 1.

28 M. M. M. Carrijo, H. Lorenz, C. R. Rambo, P. Greil and N. Travitzky, Ceram. Int., 2018, 44, 8116-8124.

29 H. Wang, J. Qian, H. Li and F. Ding, Prog. Org. Coat., 2018, 120, 19-27.

30 W. Yao, Q. Tian, J. Liu, et al., Nanoscale, 2017, 9, 1598215989.

31 M. You, J. Zhong, Y. Hong, Z. Duan, M. Lin and F. Xu, Nanoscale, 2015, 7, 4423-4431.

32 J. Andres, R. D. Hersch, J.-E. Moser and A.-S. Chauvin, Adv. Funct. Mater., 2014, 24, 5029-5036.

33 M. Kurek, I. E. Garofulić, M. T. Bakić, M. Ščetar, V. D. Uzelac and K. Galić, Food Hydrocolloids, 2018, 84, 238-246.

34 Z. Żołek-Tryznowska, J. Izdebska and M. Gołąbek, Color. Technol., 2014, 130, 314-318. 